\title{
Jan und Hein und KLaas und Pit
}

Mythos Universität

»Alle, die mit uns auf Kaperfahrt gehen, müssen Männer mit Bärten sein«. Übersetzt heißt das: Es dürfen erstens keine Frauen und zweitens keine Männer ohne Bärte, also keine Knaben, sein, auf der Kaperfahrt, da, wo es so richtig kernig und ernsthaft zugeht. Das scheint auch in der (deutschen) Wissenschaft so zu sein: Bei ihr sieht es ja - trotz Aleida Assmann - immer noch aus wie bei Jan und Hein und Klaas und Pit, auf diesem Schiff sind immer noch ziemlich viele Männer mit Bärten: »die haben Bärte, die fahren mit«. Sind wir in der Wissenschaft also auf Kaperfahrt, bei schwerem, gefahrvollem, männlichem Tun? Vielleicht ist das noch so im Maschinenbau oder in der Physik. Aber selbst da sind wohl kaum noch schwere Netze zu hieven, Schiffe zu entern, große Bauteile zu stemmen, riesige Geräte zu bewegen, körperliche Tätigkeiten,

Also: So ein ganz kleines Problem haben wir anscheinend doch noch mit den Frauen, wir aufgeklärten, progressiven Männer.

die dicke Armmuskeln und einen ordentlichen Bartwuchs verlangen. In den Geisteswissenschaften konnte man das mit gutem Gewissen sowieso niemals sagen, es mussten eigentlich nie Männer mit Bärten sein. Bücher lesen und schreiben konnten Frauen genauso gut; es sind ja auch zunehmend mehr Frauen dort. Und das ist auch gut so. Natürlich finden wir Männer das ganz wunderbar und haben überhaupt kein Problem damit. Wir promovieren und habilitieren Frauen (und bekommen an unserer Universität dafür sogar mehr Leistungspunkte als für das Promovieren und Habilitieren von Männern mit Bärten). Überhaupt kein Prob- 


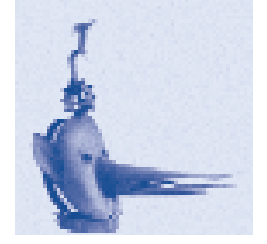

lem haben wir damit. Aber so was von überhaupt kein Problem! Oder?

Ich habe vor einigen Jahren einer interdisziplinären Forschergruppe angehört, die aus Linguisten, Soziologen, Philosophen, Literaturwissenschaftlern, Erziehungswissenschaftlern und Psychologen bestand, lauter Männern. Ohne Bedauern habe ich Frau und Kinder an zahlreichen Samstagen alleine gelassen (und die hätten mich gut gebrauchen können: zum Einkaufen, Spielen, Reparieren von Fahrrädern, Ausflügen und Ähnlichem), um mit den Kollegen interdisziplinär über Gott und die Welt, den Menschen im Allgemeinen und im Besonderen, die großen Fragen der Zeit zu diskutieren. Es war einfach herrlich. Trotz aller Terminschwierigkeiten haben wir uns gern zusammengefunden, geredet, geredet, geredet. Bis wir die Idee hatten, ein paar Frauen in die Gruppe aufzunehmen (dass vorher keine drin waren, war keine Absicht - umso schlimmer, sagt da der Dr. Freud!). Schließlich waren wir ja alle aufgeklärte, progressive Männer. Wirklich! Natürlich arbeiteten wir mit Frauen zusammen und förderten tatkräftig deren wissenschaftliche Karrieren. Warum also keine Frauen in der interdisziplinären Forschergruppe? Nun, es war das Ende der Gruppe. Es war auf einmal nicht mehr dasselbe, die Termine waren schwerer zu finden, die Familie verlangte ihr Recht - und bekam es, die eigene Publikation hatte plötzlich Vorrang vor dem interdisziplinären Gespräch. Das Ganze geriet in die Krise. Schließlich hat eine beherzte Frau den ganzen Laden gesprengt. Sie hat sich für ihr Projekt einige Männer herausgepickt, die dann natürlich keine Zeit mehr für Gott und die Welt und den Menschen im Allgemeinen und die großen Fragen der Zeit hatten. Aber es war, so scheint mir, nicht dieses Projekt, das die Gruppe spaltete und zerstörte. Der Grund war einfach, dass es nicht mehr so schön war. Also: So ein ganz kleines Problem haben wir anscheinend doch noch mit den Frauen, wir aufgeklärten, progressiven Männer.

Aber was war denn so schön am Männerverein, an Jan und Hein und Klaas und Pit?
Nun, ich glaube, es war so schön, weil es wie ganz früher war, als wir noch kleine Jungs waren, als weder der Ernst des Lebens noch Sex (was ja dasselbe ist) eine Rolle spielten. Als wir noch richtige Freunde hatten, mit denen wir spielten, uns kabbelten, die Kräfte maßen, tolle Ideen hatten und ausführten, herumspinnen konnten, den ganzen Rilke um die Wette auswendig lernten und redeten, redeten, redeten - über alles. Der Männersamstag war so schön, weil er genau das bisschen Regression erlaubte, das man braucht, um sich richtig wohl zu fühlen.

So weit die Psychologie. Vielleicht spielt auch noch die Geschichte bzw. unsere kollektive Psycho-Historie hinein: Es war vermutlich so schön, weil es so war wie dort, wo wir historisch herkommen, wir Gelehrten, wir Gottund-die-Welt-Erklärer: Es war wie in der alten Universität, das heißt wie im Kloster oder wie in der Kirche (mulier taceat in ecclesia). Die europäische Universität entwächst ja den Domschulen und Klöstern. Sie hält sich zwar für eine säkulare aufgeklärte Institution, geradezu für eine Negation des Klosters, und hat deswegen diese Vergangenheit vehement verdrängt. Aber wie jedes Verdrängte rumpelt es im Unbewussten herum, wirkt im Geheimen. Die Raum- und Organisationsformen - Zelle, Bibliothek, Refektorium, Lehr-Kanzel (wie es in Österreich heißt), Vor-Lesung, Berufung, Konvent, Kollegium - haben sowieso vieles aus den alten kirchlichen Institutionen bewahrt. Vor allem aber: Noch in Humboldts Forderung für die moderne Universität nach »Einsamkeit und Freiheit « klingt das Klösterliche nach, und im Humboldt'schen Gespräch, das die Universität ist, lebt noch das griechische ebenso wie das klösterliche Miteinanderreden der Männer fort. Einsamkeit ist nicht Zweisamkeit (mit der Frau), Freiheit ist Abwesenheit der Zwänge des wirklich ernsthaften Lebens. In den Klöstern waren weder der Ernst des Lebens noch (eigentlich) Sex (was wie gesagt dasselbe ist) zu Hause. Natürlich sind auch der Ernst des Lebens und Sex wunderbar, aber wo wir Gelehrten wirklich als Gelehrte bei uns sind, da ist 
beides eigentlich nicht. Bei den einsamen und freien Männern im Kloster wohnten der (Heilige) Geist und seine Emanation: das Wort. Das Wort, das am Anfang war, wurde gehütet, es wurde gesprochen, gehört, gesungen, gelesen, geschrieben, gedeutet. Words words words. Für den Lebensunterhalt sorgten derweil der Bruder Gärtner und für die Aufrechterhaltung des Menschengeschlechts der Bauer und der Rittersmann und deren Frauen. Gegenüber Nähr- und Wehrstand gewann der klösterliche Lehrstand durch das Wissen und

\section{Noch in Humboldts Forderung für die moderne Universität} nach"Einsamkeit und Freiheit "klingt das Klösterliche nach.

das gelehrte Sprechen gesellschaftliche Anerkennung, um den Preis des Ernstes des Lebens und der Frauen, ein hoher Preis, dem ein regressives, knabenhaftes Moment anhaftet.

Etwas von dieser alten mönchischen $\mathrm{Ge}$ meinschaft hatte die Arbeitsgruppe, etwas, das schon längst nicht mehr in der Universität zu finden war. Hier durfte wohlig an die Oberfläche, was auch wir progressiven Männer wohl nur schlecht verdrängt hatten. Deswegen war es so schön. Aber wir haben überhaupt kein Problem mit den Frauen. Echt nicht.

Aber wie geht es den Frauen? Fühlen sie sich denn wohl in diesem ehemaligen Männerkloster, das nicht wirklich in der Welt ist, mit ihren Kämpfen, ihren schweren Sachen, ihren richtigen Männern, das also bestimmt nicht die Kaperfahrt der Männer mit Bärten ist? Der Verdacht stellt sich ein, dass die Frauen vielleicht deswegen so spät in der Universität angekommen sind, weil das Universitätswesen, dieses säkularisierte Klosterwesen für nicht ganz bärtige Männer, für Frauen nicht wirklich attraktiv war - und nicht nur, weil die bösen Machos sie nicht hereingelassen haben. Die geringe Attraktivität des Klosterlebens ist vielleicht genauso verantwortlich für die späte Ankunft der Frauen im Kloster Universität wie der Widerstand der Mönche.

Holt das Klösterlich-Geistige die Frauen in der Universität ein? Ihre derzeitige Haupt- theoretikerin erklärte das Geschlecht zum kulturellen Konstrukt. Es hat gar nichts mit dem Körper zu tun (das glaubten nur noch wir Altmodler von der interdisziplinären Arbeitsgruppe). Kann sich die universitäre Frau also als Mönch entwerfen? Sind die Frauen davon begeistert? Die verbliebenen Mönche sind natürlich entzückt; sie haben es ja schon immer gesagt: Sex spielt keine Rolle. Dann wären wir endlich alle Mönche, Männer und Frauen. Nur die Frauenbeauftragte glaubt es noch nicht so ganz. Sie hat ja auch einiges zu verlieren, wenn sich das herumspricht.

Fragen Fragen Fragen. Aber sie erübrigen sich vermutlich bald, denn richtige Männer - PräsidentInnen, RektorInnen, MinisterInnen, BildungspolitikerInnen - sind ja gerade dabei, das Kloster endgültig zu schleifen. Sie haben es zunächst, wie der Marburger Philosoph Brandt in seiner Abschiedsrede auf die Universität bitter festgestellt hat, aus den repräsentativen Gebäuden in der Mitte der Stadt in lieblose Betonklötze an den Stadtrand verpflanzt (zeitgleich mit der Ankunft der Frauen an der Universität, merkwürdig, oder?). Nun aber wird es ganz und gar dem Ernst des Lebens überantwortet: Niemand soll mehr einsam und frei seinen regressiven Lüsten frönen, sich jahrelang über Bücher beugen und Wörter lesen, singen, schreiben, deuten und besprechen. Niemand, auch die Frauen nicht. Nun wird ausgebildet, berufsorientiert, BA, MA, basta (muss ja sein, ich weiß, ich weiß, schon klar, ich bin ja auch dafür, wie für die Frauen, man wird aber doch ein bisschen trauern dürfen!).

Viele ehemalige Klosterfächer, für die es keinen Beruf außerhalb der Universität gibt, werden die endgültige Säkularisierung nicht überleben. Wir - die alten Jan und Hein und Klaas und Pit - hatten das unerhörte Glück, die klösterliche Kaperfahrt noch erleben zu dürfen. Danke, Geschichte. Es gibt auch eine Gnade der frühen Geburt.

Ciao Università degli Studi, ciao bella! 